\title{
Avaliação agronômica de genótipos de amendoim na região oeste do estado de São Paulo
}

Submetido - 11 jul. $2021 \quad$ Aprovado - 31 jul. $2021 \quad$ Publicado - 30 set. 2021

http://dx.doi.org/10.52755/sas.v.2i(edesp1)141

Amenco Peanuts. E-mail: marcel.rodrigo@amenco.com.br, Tupã, SP.

Marcel Rodrigo Bazanella (i) Jair Heuert (iD

Programa de Melhoramento do Amendoim - Embrapa, Santo Antônio de Goiás, GO. E-mail: jair.heuert@embrapa.br.

Maxuel Fellipe Nunes Xavier (i)

Discente de Agronomia do IFMT Campus São Vicente - Centro de Referência de Campo Verde, Campo Verde, MT. E-mail: maxuelfellipe90@gmail.com.

Núcleo Cerrado - Embrapa Algodão Santo Antônio Nelson Dias Suassuna nelson.suassuna@embrapa.br.

Taís de Moraes Falleiro Suassuna (iD)

Programa de Melhoramento do Amendoim - Embrapa, Santo Antônio de Goiás, GO. E-mail: tais.suassuna@embrapa.br.

\section{RESUMO}

Este trabalho objetivou a avaliação agronômica de genótipos de amendoim na região oeste do estado de São Paulo. O experimento foi conduzido na área de produção comercial da Amenco Peanuts, localizado no município de Getulina-SP, na região oeste do estado de São Paulo, no ano agrícola 2020/21. Foi implantado no dia 17 de dezembro de 2020, fora do período ideal de semeadura, justamente para que fosse possível avaliar em condições extremas o potencial produtivo $e$ as características agronômicas dos genótipos. $O$ delineamento experimental utilizado foi em blocos casualizados, com quatro repetições. Os tratamentos foram treze genótipos de amendoim, sendo onze linhagens (2091 OL, 2133 OL, 1253 OL, 2131 OL, 2110 OL, 2136 OL, 2010 OL, 2101 OL, 2056 OL, 1876 OL e 2250 OL), uma cultivar (BRS 423 OL), todas desenvolvidas pelo Programa de Melhoramento do Amendoim da Embrapa e uma cultivar argentina da El Carmen (Granoleico). As parcelas eram constituídas por duas linhas de três metros de comprimento, com espaçamento de $0,90 \mathrm{~m}$ e área da parcela de $5,4 \mathrm{~m}^{2}$. Foram avaliadas severidade de mancha preta, massa de 100 grãos e produtividade de vagens. Com base no presente estudo de campo, conclui-se que as menores notas de severidade foram obtidas com os genótipos BRS 423 OL e 2091 OL. As maiores massas de 100 grãos foram constatadas com o 2010 OL e 1253 OL. Por fim, as maiores produtividades foram obtidas com 2010 OL, 2110 OL, 2101 OL, BRS 423 OL, 2136 OL, 1253 OL e 2133 OL.

Palavras-chave: Arachis hypogaea L.; Programa de Melhoramento; Produtividade.

\section{Agronomic evaluation of peanut genotypes in the western region of the state of São Paulo}

\section{ABSTRACT}

This work aimed at the agronomic evaluation of peanut genotypes in the western region of the state of São Paulo. The experiment was conducted in the commercial production area of Amenco Peanuts, located in the city of Getulina-SP, in the western region of the state of São

Este é um trabalho de acesso aberto e distribuído sob os Termos da Creative Commons Attribution Attribution-NonCommercial-ShareAlike 4.0 International. 
Paulo, in the agricultural year 2020/21. It was implemented on December 17, 2020, outside the ideal sowing period, together so that it was possible to evaluate the productive potential and the agronomic characteristics of the genotypes under extreme conditions. The experimental design used was in randomized blocks, with four replications. The treatments were thirteen peanut genotypes, eleven lines (2091 OL, 2133 OL, 1253 OL, 2131 OL, 2110 OL, 2136 OL, 2010 OL, 2101 OL, 2056 OL, 1876 OL and 2250 OL), one cultivar (BRS 423 OL), all developed by Embrapa's Peanut Improvement Program and an Argentine cultivar by EI Carmen (Granoleico). The plots consisted of two lines of three meters in length, with a spacing of $0.90 \mathrm{~m}$ and plot area of $5.4 \mathrm{~m}^{2}$. Black spot severity, 100 grain mass and pod yield were evaluated. Based on the present field study, it is concluded that the lowest severity scores were obtained with the BRS $423 O L$ and 2091 OL genotypes. The largest masses of 100 grains were found with 2010 OL and 1253 OL. Finally, the highest yields were obtained with 2010 OL, 2110 OL, 2101 OL, BRS 423 OL, 2136 OL, 1253 OL and 2133 OL.

Keywords: Arachis hypogaea L.; Improvement Program; Productivity.

\section{Evaluación agronómica de genotipos de maní en la región occidental del estado de São Paullo}

\section{RESUMEN}

Este trabajo tuvo como objetivo la evaluación agronómica de genotipos de maní en la región occidental del estado de São Paulo. El experimento se realizó en el área de producción comercial de Maní Amenco, ubicada en la ciudad de Getulina-SP, en la región occidental del estado de São Paulo, en el año agrícola 2020/21. Se implementó el 17 de diciembre de 2020, fuera del período ideal de siembra, en conjunto para que fuera posible evaluar el potencial productivo y las características agronómicas de los genotipos en condiciones extremas. El diseño experimental utilizado fue en bloques al azar, con cuatro repeticiones. Los tratamientos fueron trece genotipos de maní, once líneas (2091 OL, 2133 OL, 1253 OL, 2131 OL, 2110 OL, 2136 OL, 2010 OL, 2101 OL, 2056 OL, 1876 OL y 2250 OL), un cultivar (BRS 423 OL), todos desarrollados por el Programa de Mejoramiento de Maní de Embrapa y un cultivar argentino de El Carmen (Granoleico). Las parcelas constaban de dos líneas de tres metros de longitud, con una distancia de 0,90 m y una superficie de parcela de 5,4 $\mathrm{m}^{2}$. Se evaluó la severidad de la mancha negra, la masa de 100 granos y el rendimiento de las vainas. Con base en el presente estudio de campo, se concluye que los puntajes de severidad más bajos se obtuvieron con los genotipos BRS 423 OL y 2091 OL. Las mayores masas de 100 granos se encontraron con 2010 OL y 1253 OL. Finalmente, los mayores rendimientos se obtuvieron con 2010 OL, 2110 OL, 2101 OL, BRS 423 OL, 2136 OL, 1253 OL y 2133 OL.

Palabras clave: Arachis hypogaea L.; Programa de Mejoramiento; Productividad.

\section{Introdução}

As culturas da soja e milho possuem significância na cadeia produtiva do agronegócio brasileiro, onde estimativas apontam para uma produção recorde de 262 milhões de toneladas um crescimento de $2 \%$ compara a safra anterior. A safra do amendoim no período 2020/21, projetase uma produção de 597 mil toneladas, com crescimento de $7 \%$ maior que na última safra (CONAB, 2021). 
O estado de São Paulo possui destaque como o maior produtor da cultura do amendoim, com sua produção destinada principalmente para comercialização como matéria prima (indústria de alimentos, fabricação de óleo e exportação) e consumo in natura. Dentro do estado algumas regiões se destacam, a exemplo da região Oeste, que nos últimos anos obteve significativos investimentos em infraestrutura nas etapas de pós colheita, como na estrutura de secagem, armazenamento, beneficiamento, laboratórios de análises de qualidade e a industrialização para exportação in natura e fabricação do óleo bruto. Além disso, a indústria de confeitos de doces, salgados e snacks, se localiza nas proximidades da região produtora.

Nesta safra de 2020/21, a semeadura do amendoim que geralmente ocorre a partir do mês de outubro teve que ser adiada para os meses de novembro e dezembro, por conta de déficit hídrico, ou seja, falta de chuva em diferentes áreas de cultivo de São Paulo. Com base nisso, a CONAB (2021) retrata que tais adversidades na época de semeadura podem interferir na produtividade média da cultura nesta safra. Este trabalho objetivou a avaliação agronômica de genótipos de amendoim na região oeste do estado de São Paulo.

\section{Material e métodos}

O experimento foi conduzido na área de produção comercial da Amenco Peanuts, localizado no município de Getulina-SP, na região oeste do estado de São Paulo, no ano agrícola 2020/21. Foi implantado no dia 17 de dezembro de 2020, fora do período ideal de semeadura, justamente para que fosse possível avaliar em condições extremas o potencial produtivo dos genótipos.

O município de Getulina, na região oeste estado de São Paulo, localiza-se geograficamente na latitude $21^{\circ} 47^{\prime} 55^{\prime \prime}$ sul, longitude $49^{\circ} 55^{\prime} 43^{\prime \prime}$ oeste, e altitude de 487 metros. Os tipos de solos comuns são o Latossolo Vermelho-Escuro distrófico e o Argissolo Vermelho-Amarelo Tb. Quanto a climatologia, as médias de temperatura são de máxima de $30 \stackrel{\circ}{ } \mathrm{C}$ e mínima de $15 \stackrel{\circ}{\circ}$. A precipitação pluviométrica anual é de $1.370 \mathrm{~mm}$. A região é 
classificada como Cwa, mesotérmico, sendo tropical de altitude, com inverno seco e chuvas de verão (ECO, 2015; KÖPPEN; GEIGER, 1928).

O delineamento experimental utilizado foi em blocos casualizados, com quatro repetições. Os tratamentos foram treze genótipos de amendoim, sendo onze linhagens (2091 OL, 2133 OL, 1253 OL, 2131 OL, 2110 OL, 2136 OL, 2010 OL, 2101 OL, 2056 OL, 1876 OL e 2250 OL), uma cultivar (BRS $423 \mathrm{OL}$ ), todas desenvolvidas pelo Programa de Melhoramento do Amendoim da Embrapa e uma cultivar argentina da El Carmen (Granoleico). As parcelas foram constituídas por duas linhas de semeadura de três metros de comprimento, com espaçamento de $0,90 \mathrm{~m}$. A área total da parcela foi de $5,4 \mathrm{~m}^{2}$.

O manejo de correção da fertilidade do solo e manejo fitossanitário foram realizados conforme as recomendações para a cultura. A densidade de plantas foi de 17 plantas. $\mathrm{m}^{-1}$. Avaliação de severidade de mancha preta (Cercosporidium personatum), foi avaliada usando uma escala diagramática da incidência com notas de 1 a 9, em que 1 indicava a planta sem doença e 9 desfolha intensa (SUBRAHMANYAM et al., 1982).

A colheita foi realizada aos 130 dias após o plantio. A produtividade de vagens ( $\mathrm{kg}^{-\mathrm{ha}^{-1}}$ e sacas.alqueire $\left.{ }^{-1}\right)$ foi avaliada mediante a colheita de dois metros centrais das duas linhas, totalizando $5,4 \mathrm{~m}^{2}$. A massa de 100 grãos $(\mathrm{g})$, foi realizada mensurada em laboratório com balança digital, após a debulha mecanizada das vagens colhidas. Os dados foram submetidos à análise de variância pelo Teste $\mathrm{F}$ e as médias dos tratamentos foram comparados pelo teste de Scott-Knott, no nível de $p \geq 0,05$ de probabilidade, por meio do programa computacional SISVAR 5.6 (FERREIRA, 2019).

\section{Resultados e discussão}

Observa-se diferença significativa para a severidade de mancha preta (notas), massa de 100 grãos (g) e produtividade de vagens (kg.ha-1) em função de diferentes genótipos de amendoim na região da Alta Paulista, nas condições de Getulina-SP. As menores notas de severidade foram obtidas nos genótipos BRS 423 OL $(5,7)$ e 2091 OL (6,0) (Tabela 1). 
Concordando com os dados obtidos por Zammataro et al. (2020), que constataram notas semelhantes com os genótipos 2101 OL $(8,2)$, Granoleico $(7,9), 2136$ OL $(7,8)$ e 2133 OL $(7,3)$, nas condições de Parapuã-SP, na região da Alta Paulista.

Tabela 1. Severidade de mancha preta (notas), massa de 100 grãos (g) e produtividade de vagens ( $\mathrm{kg} \cdot \mathrm{ha}^{-1}$ e sacas.alqueire ${ }^{-1}$ ) em função de diferentes genótipos de amendoim na região oeste do estado de São Paulo. GetulinaSP, 2020/21.

\begin{tabular}{|c|c|c|c|c|}
\hline \multirow{2}{*}{ Genótipos } & \multirow{2}{*}{$\begin{array}{c}\text { Severidade } \\
\text { (notas) }\end{array}$} & \multirow{2}{*}{$\begin{array}{c}\text { Massa de } \\
100 \text { grãos } \\
\text { (g) }\end{array}$} & \multicolumn{2}{|c|}{ Produtividade de vagens } \\
\hline & & & (kg.ha-1) & ${\text { (sacas.alqueire }{ }^{-1} \text { ) }}$ \\
\hline 2010 OL & $7,5 \mathrm{~b}$ & $78,1 \mathrm{a}$ & $3.822,0 \mathrm{a}$ & 370,0 \\
\hline $2110 \mathrm{OL}$ & $7,4 \mathrm{~b}$ & $74,2 \mathrm{c}$ & $3.419,8 \mathrm{a}$ & 331,1 \\
\hline $2101 \mathrm{OL}$ & $8,1 \mathrm{a}$ & $76,0 \mathrm{~b}$ & $3.367,3 \mathrm{a}$ & 326,0 \\
\hline BRS $423 \mathrm{OL}$ & $5,7 \mathrm{c}$ & $75,9 \mathrm{~b}$ & $3.325,8 a$ & 322,0 \\
\hline $2136 \mathrm{OL}$ & $7,5 b$ & $73,4 d$ & $3.311,8 a$ & 320,6 \\
\hline $1253 \mathrm{OL}$ & $7,1 \mathrm{~b}$ & $78,0 \mathrm{a}$ & $3.207,0 \mathrm{a}$ & 310,5 \\
\hline $2133 \mathrm{OL}$ & $7,0 \mathrm{~b}$ & $76,3 \mathrm{~b}$ & $3.159,9 a$ & 305,9 \\
\hline 2091 OL & $6,0 \mathrm{c}$ & $72,6 d$ & $2.572,8 \mathrm{~b}$ & 249,1 \\
\hline $1876 \mathrm{OL}$ & $8,3 \mathrm{a}$ & $72,9 d$ & $2.360,5 \mathrm{~b}$ & 228,5 \\
\hline $2250 \mathrm{OL}$ & $8,6 \mathrm{a}$ & $74,7 \mathrm{c}$ & $2.215,2 \mathrm{~b}$ & 214,5 \\
\hline $2131 \mathrm{OL}$ & $7,3 \mathrm{~b}$ & $75,5 b$ & $2.136,8 b$ & 206,9 \\
\hline $2056 \mathrm{OL}$ & $8,1 \mathrm{a}$ & $75,4 \mathrm{~b}$ & $2.031,9 \mathrm{~b}$ & 196,7 \\
\hline Granoleico & $7,6 \mathrm{~b}$ & $71,9 \mathrm{e}$ & $1.827,0 \mathrm{~b}$ & 176,9 \\
\hline Média & 7,4 & 75,0 & $2.827,5$ & 273,7 \\
\hline C.V. (\%) & 6,5 & 0,7 & 12,6 & - \\
\hline $\mathrm{Pr}>\mathrm{FC}$ & $>0,0001^{*}$ & $>0,0001^{*}$ & $0,0001^{*}$ & - \\
\hline
\end{tabular}

$\mathrm{Na}$ massa de 100 grãos, verifica-se as maiores massas nos genótipos 2010 OL $(78,1 \mathrm{~g})$ e $1253 \mathrm{OL}(78,0 \mathrm{~g})$. Por outro lado, a menor foi obtida no Granoleico (71,9 g) (Tabela 1). Corroborando com Uitdewilligen et al. (2020), que obtiveram massa correlata com o BRS $423 \mathrm{OL}$ (76,5 g), nas condições de Jaboticabal-SP, na região da Alta Mogiana. Nesse sentido, Zammataro et al. (2020), observaram massas semelhantes com os genótipos 2101 OL (78,7 g), 2056 OL (77,9 g), 2133 OL (76,8 g) e 2136 OL $(75,5 \mathrm{~g})$.

As maiores produtividades de vagens, em ordem decrescente, foram observadas nos genótipos 2010 OL (3.822,0 kg.ha-1), 2110 OL (3.419,8 
kg.ha-1), 2101 OL (3.367,3 kg.ha-1), BRS 423 OL (3.325,8 kg.ha-1), 2136 OL (3.311,8 kg.ha-1), $1253 \mathrm{OL}\left(3.207,0 \mathrm{~kg} \cdot \mathrm{ha}^{-1}\right)$ e $2133 \mathrm{OL}\left(3 \cdot 159,9 \mathrm{~kg} \cdot \mathrm{ha}^{-1}\right)$. No entanto, os genótipos $2091 \mathrm{OL}\left(2.572,8 \mathrm{~kg} \cdot \mathrm{ha}^{-1}\right), 1876 \mathrm{OL}$ (2.360,5 kg.ha-1), $2250 \mathrm{OL}\left(2.215,2 \mathrm{~kg} \cdot \mathrm{ha}^{-1}\right), 2131 \mathrm{OL}\left(2.136,8 \mathrm{~kg}^{-\mathrm{ha}^{-1}}\right), 2056 \mathrm{OL}(2.031,9$ kg.ha-1) e Granoleico (1.827,0 $\left.\mathrm{kg}^{-1} \mathrm{ha}^{-1}\right)$, apresentaram as menores produtividades do presente estudo (Tabela 1).

Bazanella et al. (2020), obtiveram produtividades similares as obtidas com os genótipos BRS 423 OL (3.378,4 kg.ha-1) e 1253 OL (2.944,1 kg.ha-1), nas condições de Tupã-SP, na região da Alta Paulista. Por outro lado, ressalta-se que somente o genótipo $2010 \mathrm{OL}$ (3.822,0 kg.ha-1), alcançou produtividade superior à estimativa média nacional $(3.679,0$ kg.ha1) de primeira safra da CONAB (2021).

Possivelmente, os níveis elevados de incidência de $C$. personatum podem ter contribuído para o baixo nível produtivo, mesmo para os genótipos BRS 423 OL e 2091 OL, para os quais se constataram menores notas de severidade da doença, em relação aos demais tratamentos. Assim, as produtividades obtidas não superaram a média nacional (CONAB, 2021) e aos estudos de Bazanella et al. (2020) e Zammataro et al. (2020). Ademais, o plantio tardeio, na data de 17 de dezembro de 2020, pode ter influenciado nos níveis de produtividade obtidos.

\section{Conclusões}

Com base no presente estudo de campo, conclui-se que as menores notas de severidade de mancha preta foram obtidas com os genótipos BRS $423 \mathrm{OL}$ e $2091 \mathrm{OL}$. As maiores massas de 100 grãos foram constatadas com o 2010 OL e o 1253 OL. Por fim, as maiores produtividades foram obtidas com 2010 OL, 2110 OL, 2101 OL, BRS 423 OL, 2136 OL, 1253 OL e 2133 OL.

\section{Agradecimentos}

Os autores agradecem à Amenco Peanuts pela cooperação técnica, suporte financeiro e aos produtores Antônio Fernandes e Ricardo 
da Silveira Fernandes, pela disponibilidade da área para realização do experimento em Getulina-SP. 


\section{Referências}

BAZANELLA, M. R.; HEUERT, J.; RIBEIRO, R. P.; SUASSUNA, T. M. F.; ZAMMATARO, V. H. L. Desempenho agronômico de genótipos de amendoim sob alta severidade de mancha anelar na Alta Paulista. South American Sciences, v. 1, n. 1, p. e2006, 2020.

CONAB. Acompanhamento da Safra Brasileira de Grãos. Safra 2020/21 Sétimo levantamento, v. 8, n. 7, p. 1-117, 2021. Disponível em: <https://www.conab.gov.br/info-agro/safras>. Acesso em: 26 abr. 2021.

ECO Consultoria Ambiental e Comércio Ltda. Plano de Gerenciamento Integrado de Resíduos Sólidos de Getulina (SP). Getulina-SP: Relatório ECO 159/15. 130 p.

FERREIRA, D. F. SISVAR: um sistema de análise de computador para efeitos fixos projetos de tipo de partida dividida. Revista Brasileira de Biometria, v. 37, n. 4, p. 529-535, 2019.

KÖPPEN, W.; GEIGER, R. Klimate der Erde. Gotha: Verlagcondicionadas. Justus Perthes. 1928.

SUBRAHMANYAM, P.; MCDONALD, D.; GIBBONS, R. W.; NIGAM, S. N.; NEVILL, D. J. Resistance to rust and late leaf spot diseases in some genotypes of Arachis hypogaea. Peanut Science, v. 9, p. 9-14, 1982.

UITDEWILLIGEN, G. S.; LIBANORE, F. M.; HEUERT, J.; MARTINS, K. B. B.; SUASSUNA, T. M. F. Desempenho agronômico de cultivares de amendoim na região da Alta Mogiana. South American Sciences, v. 1, n. 1, p. e2015, 2020.

ZAMMATARO, V. H. L.; HEUERT, J.; RODRIGUES, L. L.; DANELUTTI, M. L.; SUASSUNA, T. M. F. Desempenho agronômico de genótipos de amendoim na região da Alta Paulista. South American Sciences, v. 1, n. 1, p. e2005, 2020. 